\title{
VASCULAR AND PERITONEAL ACCESS FOR DIALYSIS - CRITERIA
}

\author{
Staykova S. \\ Clinic of Dialysis, St. Marina University Hospital of Varna
}

\begin{abstract}
The creation and maintenance of functioning vascular access along with the associated complications constitute the most common cause of morbidity, hospitalization, and cost in patients with end-stage renal disease. Vascular access for dialysis can be accomplished via arteriovenous fistula or percutaneous catheter. A peritoneal access can be made use of, too. An organized monitoring approach that includes regular assessment of the clinical parameters of the access and adequacy of the dialyses should be implemented in every dialysis centre. Such a proactive approach can be expected to reduce the incidence of thrombosis and increase patency. Data should be tabulated and tracked within each dialysis centre as part of a quality assurance/continuous quality improvement (QA/CQI) programme. Central vein cannulations can be carried out under ultrasonographic guidance, too. In fact, the NFK-DOQI committee recommends routine real-time ultrasound-guided insertion to reduce insertion-related complications.
\end{abstract}

Key words: vascular access device, arteriovenous fistulas, percutaneous catheters, peritoneal access, monitoring

\section{INTRODUCTION}

The number of patients with end-stage renal disease (ESRD) in the United States has increased steadily since 1980 onwards. Its prevalence among the population in this country has quintupled since 1980 , from 271 to nearly $1,400 /$ million. It is estimated that by 2030 , there may be 2,240 million ESRD patients. Almost $60 \%$ of them are expected to be with diabetes mellitus, half to be 65 years or older, and half to be nonwhite.

Extracorporeal blood dialysis was introduced in 1943 by Kolff and colleagues. However, application of this approach was hindered by the requirement for repeated and routine access to the circulation.

\section{Vascular access via arteriovenous fistula}

The full potential of hemodialysis for patient salvage was realized only after the introduction of the external arteriovenous (AV) shunt by Quinton et al. in (1960) and of the endogenous AV fistula (AVF) by Brescia et al. in 1966 (1-4). The subsequent introduction of synthetic vascular prostheses (AVG) has permitted continued access in patients with exhausted peripheral venous sites $(6,7)$. Vascular access via AVF and AVG is show on Fig. 1 and Fig. 2.

\footnotetext{
Address for correspondence

S. Staykova, Clinic of Dialysis, St. Marina University Hospital of Varna, Medical University of Varna

55 Marin Drinov Str.

Phone: 0885/841 663

e-mail: svetlastaykova@abv.bg
}

However, the long-term performance of such prostheses remains inferior to that of autogenous fistulas. The creation and maintenance of functioning vascular access along with the associated complications constitute the most common cause of morbidity, hospitalization, and cost in ESRD patients.

The ideal vascular access route permits a flow rate that is adequate for the dialysis prescription ( $\mathrm{Qb} 300 \mathrm{~mL} / \mathrm{min})$, can be used for extended periods, and has a low complication rate. The native AVF remains the gold standard. In 1997, the National Kidney Foundation Dialysis Outcome and Quality Initiative (NKF-DOQI) (1,8) organized multidisciplinary work groups for evaluation of any data about vascular access available and concluded that quality of life and overall outcome could significantly be improved for hemodialysis patients if two primary goals were achieved: i) increased placement of native AVFs: a minimum of $50 \%$ of the new dialysis patients should have primary AVFs and ii) detection of dysfunctional access before thrombosis of the access route occurs.

This report contains clinical practice guidelines on all aspects of vascular access and was updated in 2001.

\section{Outcome evaluation}

Thrombotic events are the leading cause of access loss. They result mainly from venous outflow stenosis that can be detected and treated before thrombosis occurs (Fig. 3, Fig. 4). An organized monitoring approach that includes regular assessment of the clinical parameters of the access and of the adequacy of the dialysis should be implemented in every dialysis centre $(6,10)$. Such a proactive approach 


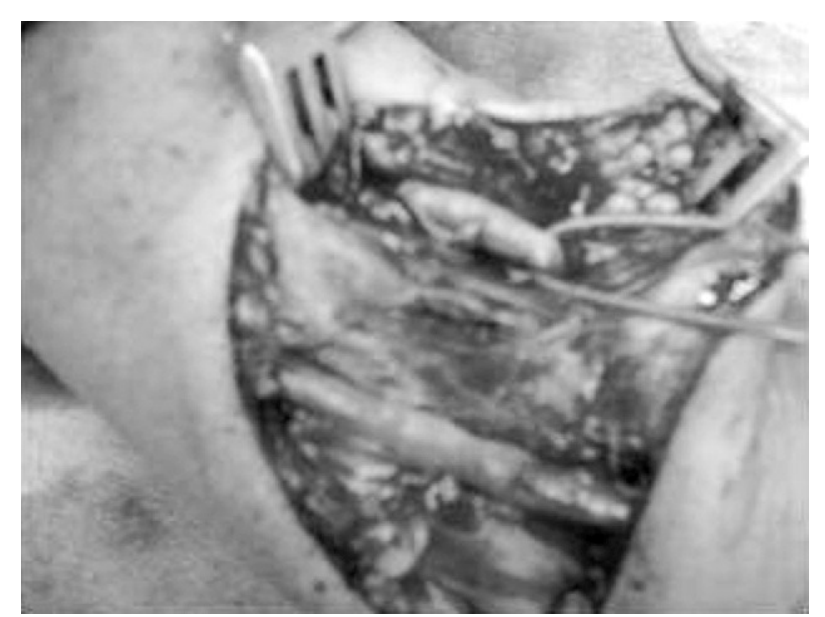

Fig. 1. AVF image

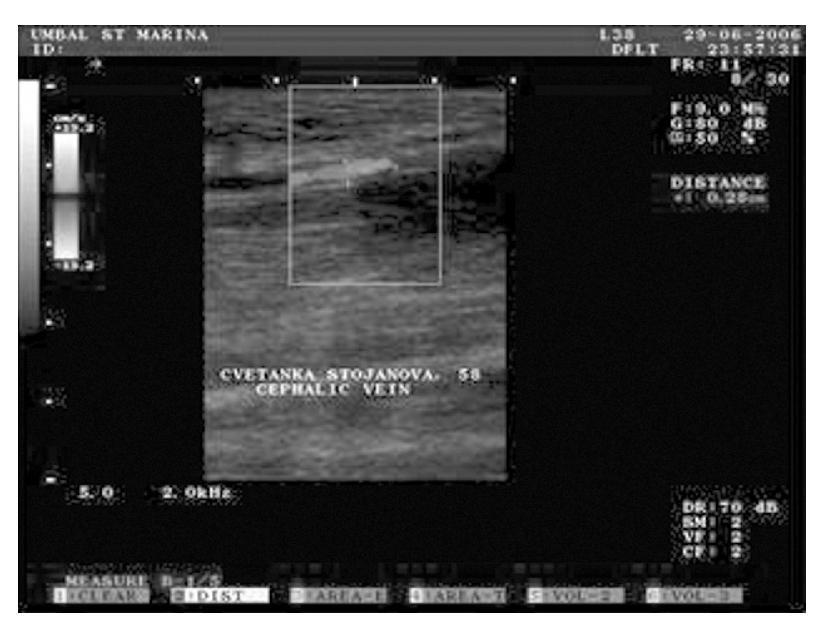

Fig. 3. Venous outflow stenosis

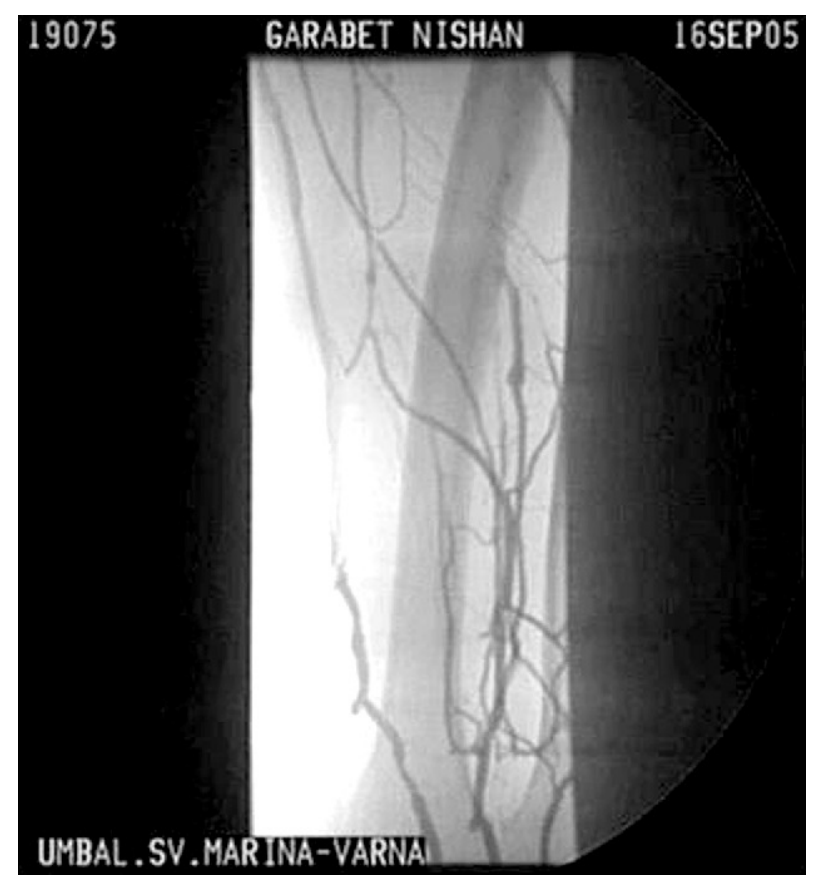

Fig. 4. Percutaneous transluminal angioplasty

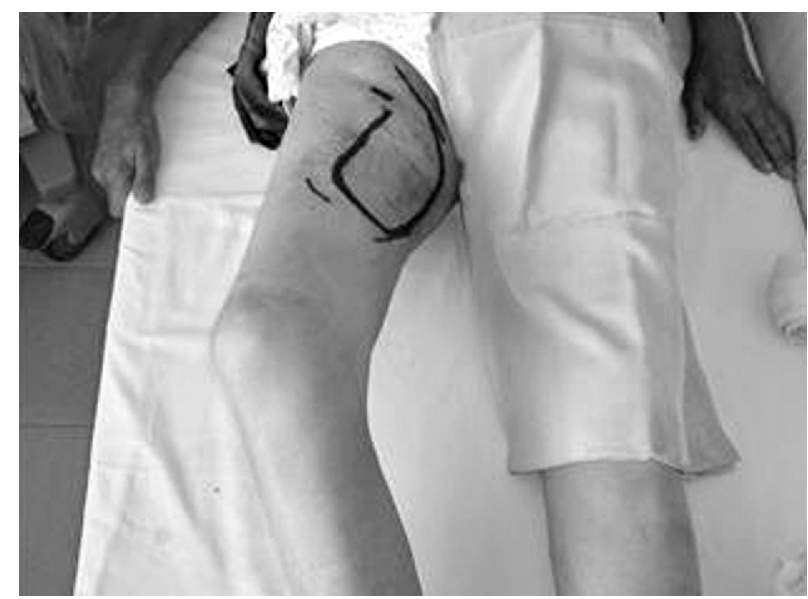

Fig. 2. AVG image

could reduce the incidence of thrombosis and increase patency.

Physical examination of the AVG or autogenous AVF should be performed weekly and should include not only inspection and palpation for changes in the physical characteristics of the pulse or thrill but also a search for indirect signs of graft dysfunction (e.g., arm swelling, prolonged bleeding after needle withdrawal and aneurysm or pseudoaneurysm). In addition to physical examination, the NKF-DOQI committee recommends routine access monitoring at least monthly with one or more of the following techniques:

1. Intra-access flow assessment using Doppler flow (Fig. 5), magnetic resonance imaging, or ultrasound dilution online during dialysis. A trend towards decreasing flow or a flow rate lower than $600 \mathrm{~mL} / \mathrm{min}$ for an AVG and lower than 200 to $300 \mathrm{~mL} / \mathrm{min}$ for an autogenous AVF is predictive of a high likelihood of access stenosis and eventual thrombosis.

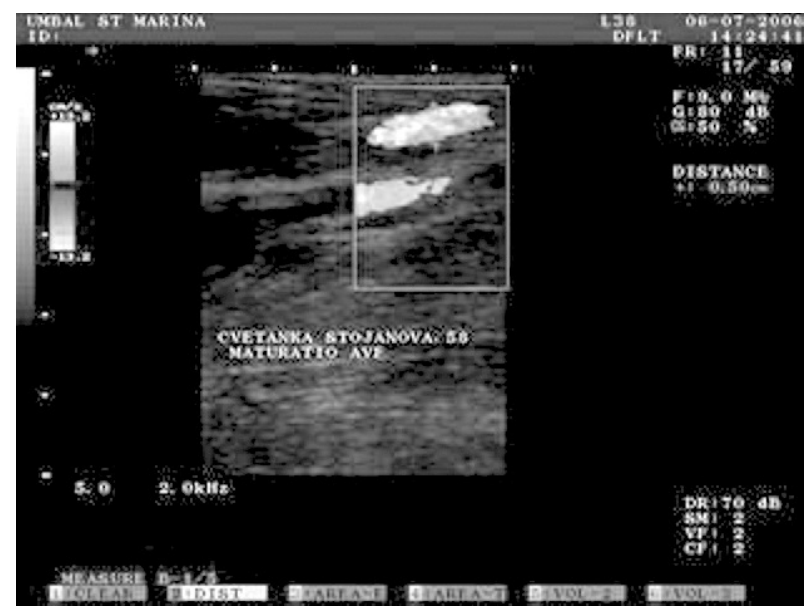

Fig. 5. Dupplex Doppler image

2. Static or dynamic venous pressure measurement. Progressively increasing pressures or pressures that exceed the threshold value determined by each centre's 
own protocol are predictive of significant venous outlet stenosis.

3. Urea and nonurea recirculation measurement. Recirculation is defined as the percentage of flow that is recirculated from the venous line into the dialyser inflow by retrograde blood flow through the fistula. A recirculation value exceeding $10 \%$ to $20 \%$ is significant and, usually, indicates low arterial blood flow or venous stenosis.

4. Delivered dialysis dose. Its decrease is associated with venous outflow stenosis.

5. Arterial prepump pressure. An elevated negative pressure is a sign of inflow insufficiency.

Data should be tabulated and tracked within each dialysis centre as part of a quality assurance/continuous quality improvement (QA/CQI) programme.

\section{Vascular access via percutaneous catheter}

Percutaneous venous catheterization is a useful method of gaining immediate access to the circulation. It is, however, associated with significant risks, and the use-life of this type of access is shorter than that of AVFs. Percutaneous catheterization should be reserved for patients either with acute renal failure who have an immediate need for dialysis, or with chronic renal failure if no permanent vascular or peritoneal access route can be established or if it has not matured yet. Permanent catheters have been used for as long as 4 years, but long-term follow-up data on large numbers of patients are lacking $(5,9)$. Most patients readily accept this approach to dialysis: cuffed catheters allow the so-called no-needle dialysis with high flow rates, they eliminate the problem of vascular steal and do not exert any effect on cardiac function. In addition, patients appreciate that the access site is hidden from view, it does not limit physical activity and thus it does not require any special care between treatments.

Puncture sites of choice are internal jugular vein and subclavian venous approach (Fig. 6).

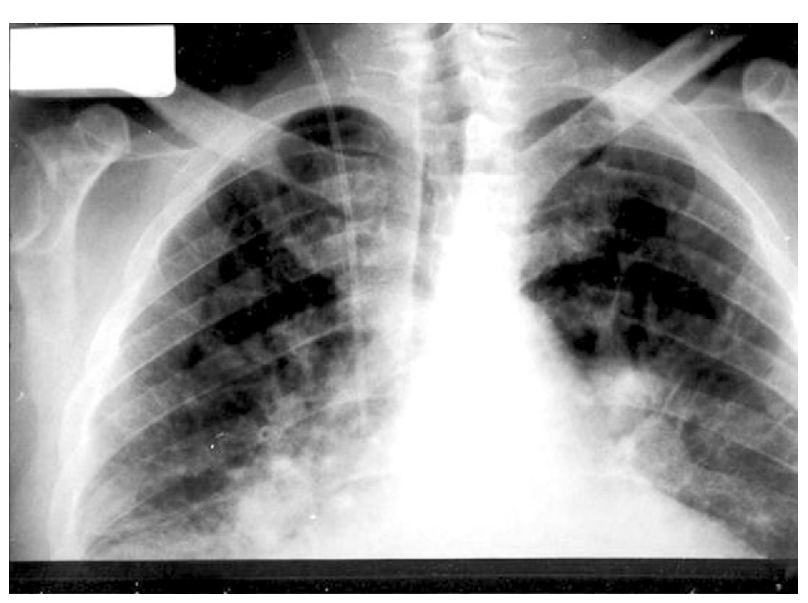

Fig. 6. Jugular central vascular access

Vein cannulation can be carried out under ultrasonographic guidance, too (5). In fact, the NFK-DOQI committee rec- ommends routine real-time ultrasound-guided insertion to reduce insertion-related complications.

\section{Outcome evaluation}

To improve dialysis outcomes and to maintain or improve quality of care for dialysis patients, percutaneous catheterization should be performed in selected cases only. Taking into consideration that this procedure is associated with higher complication rates, morbidity, and mortality than AV fistulization, the goal should be to use percutaneous catheters in fewer than $10 \%$ of hemodialysis patients requiring long-term vascular access. In those cases in which central catheter placement is the best option available, according to the NFK-DOQI, usage of a consistent technique by skilled operators should reduce the risk of serious complications necessitating intervention down to $2 \%$ or lower.

\section{Peritoneal access}

It is estimated that approximately $20 \%$ of transfers from peritoneal dialysis to hemodialysis are secondary to a catheter-related problem $(9,10)$. It is, therefore, essential that the insertion of peritoneal dialysis catheters be performed by a competent and experienced team. In fact, despite the development of new insertion techniques and the availability of increasingly sophisticated catheters, the quality of the surgical procedure and the postoperative care remains the most important prognostic factor. Analysis of the different implantation protocols described in the literature shows that the so-called centre effect is, in fact, a remarkable confounding factor. Regardless of the technique used, the catheter survival rate should be at least $80 \%$ at one year.

\section{REFERENCES}

1. Montreulle, B., S. Staykova, B. Denchev. Vascular and peritoneal dialysis access - TASK criteria. Varna, Steno, 2006. 71 p. (in Bulgarian).

2. Kraev, Z. Vascular access. Peritoneal dialysis. Sofia, Paradigma, 2008 (in Bulgarian).

3. Brescia, M. J., J. E. Cimino, K. Appell, B. J. Hurwich, B. H. Scribner. Chronic hemodialysis using venipuncture and a surgically created arteriovenous fistula.- J. Am. Soc. Nephrol., 10, 1999, No 1, 193-199.

4. Cueto-Manzano, A. M., E. Rojas-Campos. Status of renal replacement therapy and peritoneal dialysis in Mexico.- Perit. Dial. Int., 27, 2007, No 2, 142-148.

5. Haire, W. D., T. G. Lynch, R. P. Lieberman, J. A. Erdney. Duplex scans before subclavian vein catheterization predict unsuccessful catheter placement.- Arch. Surg., 127, 1992, No 2, 229-230.

6. Montreuil, B. Vascular and peritoneal access: Introduction. ACS Surgery Online. WebMD Inc, 2002.

7. National Kidney Foundation DOQI: clinical practice guidelines for hemodialysis vascular access.- Am. J. Kidney Dis., 30, 1997, Suppl. 3, S150. 
8. Prevention of peritonitis in CAPD.- Lancet, $\mathbf{3 3 7}$, 1991, p. 22.

9. Quinton, W., D. Dillard, B. H. Scribner. Cannulation of blood vessels for prolonged hemodialysis.- Trans. Am. Soc. Artif. Intern. Organs, 6, 1960, 104-113.
10. Walters, B. A. J., P. Pennell, J. P. Bosch. Quality assurance and continuous quality improvement programs for vascular access care.- Contrib. Nephrol., 142, 2004, 323-349. 